\title{
Biochemical Studies on Garbohydrates.
}

\author{
CX. Hexosamine Compounds in Lung.
}

First Report.

By

\section{Motoyuki Utusi.}

(宇㴖志 元亭)

(From the Medico-Chemical Institute, Tohoku Imperial University, Sendai. Director: Prof. H. Masamune.)

(Received for publication, August 3, 1947)

From pig lung have been isolated two glycidamins besides heparin. The present account deals with the preparation procedure and the results of chemical and biological tests.

\section{EXPERIMENTAL.}

Preparation Procedure and Chemical Properties of the Products.

Fresh pig lungs were removed mechanically from bronchioles and the adhering pleural membrane as perfectly as possible, washed from blood stains and minced. The tissue pulp was placed in 5 volumes of $95 \%$ alcohol for several days, squeezed and again dehydrated with 3 volumes of the alcohol similarly. It was followingly boiled twice with 10 volumes of the alcohol for 2 hours and dried in a Faust apparatus. $500 \mathrm{gm}$. of the dried material obtained thus from $2500 \mathrm{gm}$. of the fresh pulp were then agitated in 5 volumes of $5 \% \mathrm{NaCl}$ solution for 2 days, using thymol $(0.5 \mathrm{cc}$. of its $25 \%$ alcoholic solution for every $100 \mathrm{cc}$. of the $\mathrm{NaCl}$ solution) as a preservative. The extract was percolated and cen. trifuged. The turbid supernatant liquid were precipitated ${ }^{11}$ by acidifying to $\mathrm{pH} 4.5$ with $30 \%$ acetic acid. Centrifuged again. The clear liquid obtained was neutralized with $2 \% \mathrm{NaOH}$ solution, dialyzed (cellophane membrane) against running water for 3 days, and some precipitate, which appeared thereby, having been removed, distilled under reduced pressure to about $10 \mathrm{cc}$. 3 volumes of $95 \%$ alcohol and a little sodium acetate were added and the deposit washed with $95 \%$ alcohol and ether and dried in a vacuum desiccator $\left(\mathrm{CaCl}_{2}\right) .0 .9 \mathrm{gm}$. was the yield. A repeated extraction of the residue gave $0.4 \mathrm{gm}^{2}{ }^{2}$ 
The products combined were taken up in $10 \mathrm{cc}$. of water, and a small part undissolved was centrifuged off, then the liquid was acidulated, removed from the acid-insoluble substance ${ }^{3}$ and precipitated after neutralization as above. The dried substance weighed $1.1 \mathrm{gm}$. and was positive in biuret, Molisch (violet), aniline acetate (for pentose and hexuronic acid), Neuberg and Saneyoshi naphthoresorcinol (for hexuronic acid), Osaki and Turumi (both by the direct and indirect processes), barium sulfate (for hydrolysable sulfur) and ammonium molybdate (for phosphorus) tests, which suggested our having a mixture of proteins, nucleic acid, heparin and other hexosamine compounds. Hence, removal of the usual proteins (made up of almost merely amino acids) was carried out prior to fractionation as follows. The substance was at first kneaded with a little glycol to a dough, stirred up with the solvent that had been increased to $10 \mathrm{cc}$. and subjected to prolonged centrifugation. The transparent supernatant fluid was precipitated with 2 volumes of acetone and a little sodium acetate, and the precipitate was washed with acetone (three times) and ether (twice) and dried in vacuo over $\mathrm{CaCl}_{2}$. $0.7 \mathrm{gm}$. of white powder was obtained. Extraction ${ }^{4}$ ) made again with $5 \mathrm{cc}$. of glycol gave a 0.17 gm. crop.

Preliminary fractionation. The whole product was made into a thick paste with a little water $(<1 \mathrm{cc}$.) and precipitated with $5 \mathrm{cc}$. of glacial acetic acid (99\%). Centrifuged. The centrifugate was freed from acetic acid by washing with acetone ( 4 times) and dried after substituting ether for acetone (3 changes of ether were used). On repetition of these processes phosphorus was eliminated by and by and the final phosphorusfree substance (white powder; obtained after 5 reprecipitations) amounted to $0.5 \mathrm{gm}$.-Fr. A. The supernatant liquids were united and mixed with 2 volumes of acetone and the deposit washed and dried again similar to above. $0.3 \mathrm{gm}$. (yellowish powder) was yielded here.-Fr. B. The results of qualitative tests are listed in Table I, wherefrom the transition of nucleic acid and a probable glycidamin or glycidamins into Fr. B. looks to have occurred.

Subdivision of Fr. A. This fraction $(0.5 \mathrm{gm}$.) was kneaded with $2 \mathrm{cc}$. of water, the dough weakly alkalized with drops of $2 \% \mathrm{NaOH}$ (to $\mathrm{pH} 7.2-7.4)$ and $5 \mathrm{cc}$. of $2 \%$ picric acid added to precipitate the solution. After centrifugation, the centrifugate, which was biuret-positive and gave no positive Osaki-Turumi test both by the direct and indirect processes, was discarded and the clear supernatant fluid was precipitated with acetone ( 2 volumes). The precipitate was treated first with cold acetone (three times) and then with the boiling (three times), whereby picric acid was entirely washed off, and dried up in vacuo. It was next dissolved in $20 \mathrm{cc}$. of water (dissolved clearly), and after neutralization, shaken many times 
TABLE I.

\begin{tabular}{|c|c|c|}
\hline Test & Fr. A & Fr. B \\
\hline Biuret & + & + \\
\hline$\alpha$-Naphthol- $\mathrm{H}_{2} \mathrm{SO}_{4}$ & Violet (Molisch, + ) & Blood red with violet tint \\
\hline $\begin{array}{l}\text { Aniline acetate (for pentose } \\
\text { and hexuronic acid) }\end{array}$ & + & 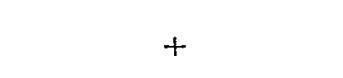 \\
\hline $\begin{array}{l}\text { Neuberg-Saneyoshi naphtho- } \\
\text { resorcinol }\end{array}$ & + & - \\
\hline $\begin{array}{l}\text { Osaki-Turumi } \\
\text { Direct } \\
\text { Indirect }\end{array}$ & $\begin{array}{l}+ \\
+\end{array}$ & + \\
\hline $\mathrm{BaSO}_{4}$ (for hydrolysable $\mathrm{S}$ ) & + & - \\
\hline Ammonium molybdate (for $\mathrm{P}$ ) & - & + \\
\hline $\begin{array}{l}\text { Ammoniacal } \mathrm{AgNO}_{3} \text { (for pu- } \\
\text { rine bases) }\end{array}$ & - & + \\
\hline
\end{tabular}

with chloroform (at first with $5 \mathrm{cc}$. and afterwards with $2 \mathrm{cc}$.), each time the gel formed being separated by centrifugation. When no more gel came out, the solution was acidified to $\mathrm{pH} 2.0$ (with $2 \% \mathrm{HCl}$ ), and deproteinization was continued using $2 \mathrm{cc}$. portions of chloroform. Finally the watery layer ${ }^{5)}$ was mixed thoroughly with $5 \mathrm{gm}$. of kaolin, and filtered under suction, then the filtrate neutralized (with $2 \% \mathrm{NaOH}$ ), distilled (under reduced pressure) to about $1 \mathrm{cc}$. and precipitated with acetone (3 volumes). The precipitate was washed with acetone and ether and dried. $0.1 \mathrm{gm}$. of white powder was yielded.-Fr. A $A_{1 \mathrm{a}}$. Kaolin on the funnel was suspended in $30 \mathrm{cc}$. of water, neutralized (with $2 \% \mathrm{NaOH}$ ) and again filtered off on a Büchner funnel. The filtrate here was also condensed and deposited as the former. Crop, $30 \mathrm{mgm}$.- $\mathrm{A}_{1 \mathrm{~b}}$. As is demonstrated in Table II, not only Fr. $A_{1 a}$ but also $A_{1 b}$ seemed heparin, although the latter was contaminated with a trace of glycidamin or of an acetylhexosamine-containing carbohydrate.

TABLE II.

\begin{tabular}{l|c|c}
\hline \multicolumn{1}{c|}{ Test } & Fr. A1a & Fr. A1b \\
\hline Biuret & - & - \\
a-Naphthol- $\mathrm{H}_{2} \mathrm{SO}_{4}$ & Green (Goldschmiedt, & ) \\
Aniline acetate & + & + \\
Neuberg-Saneyoshi & + & + \\
Osaki-Turumi & - & - \\
$\quad$ Direct & - & \pm \\
Indirect & + & +
\end{tabular}

* A faint red ring appeared neighbouring the green at the surface of contact of the two layers, and on shaking together the mixture changed light violet. 
The jellies from the neutral and acid solutions were combined, washed with water, acidified to $\mathrm{pH} 2$ (with $\mathrm{HCl}$ ), 3 times and shrunk by means of $95 \%$ alcohol. Washed with alcohol $(95 \%)$ and ether and dried. Yield, $0.15 \mathrm{gm}$.-Fr. $\mathrm{A}_{2}$. This substance was soluble in water at neutral and alkaline reactions and insoluble at $\mathrm{pH}$ 's of 4.0 and less. Tests indicated it to be a glycidamin (TAable III).

TABLE III.

\begin{tabular}{l|c}
\hline \multicolumn{1}{c|}{ Test } & Fr. $\mathrm{A}_{2}$ \\
\hline Biưret & + \\
$\alpha$-Naphthol- $\mathrm{H}_{2} \mathrm{SO}_{\mathrm{a}}$ & Violet $(\mathrm{Molisch},+)$ \\
Neuberg-Saneyoshi & - \\
Osaki-Turumi & + \\
Direct & + \\
Indirect & + \\
BaSO $_{4}$ (for hydrolysable S &
\end{tabular}

Elimination of nucleic acid from Fr. B. $0.15 \mathrm{gm}$. of this fraction was dissolved in $4 \mathrm{cc}$. of water. To the solution, which was faintly acid ( $\mathrm{pH}, 4.5)$, was added the saturated $\mathrm{Ba}(\mathrm{OH})_{2}$ in drops first until neutral and then to $\mathrm{pH} 9.4$, the precipitate appearing at each point being centrifuged off. The second supernatant liquid was acidified back to

$\mathrm{pH} 2.0$ with $3 \% \mathrm{HCl}$ and subjected to a similar procedure over again ${ }^{6)}$. Barium was removed with $3 \% \mathrm{H}_{2} \mathrm{SO}_{4}$ solution and the precipitate by 3 volumes of $95 \%$ alcohol was washed with alcohol (95\%, three times) and ether (twice) and dried in a vacuum desiccator. $90 \mathrm{mgm}$. of yellowish white powder were gained-Fr. $B_{1}$. It was water-soluble and was a glycidamin, judging from the results of reactions listed in Table IV.

Remark. In the tissue resi-

TABLE IV.

\begin{tabular}{l|c}
\hline \multicolumn{1}{c|}{ Test } & Fr. $\mathrm{B}_{1}$ \\
\hline Biuret & + \\
$\alpha$-Naphthol- $\mathrm{H}_{2} \mathrm{SO}_{4}$ & Violet $($ Molisch,+$)$ \\
Aniline acetate & - \\
Osaki-Turumi & + \\
Direct & + \\
Indirect & + \\
Ammonium molybdate & -
\end{tabular}

due; which had been exhaustively extracted with the saline solution, was still detected hexosamine (analyzed by Hamasato and Akakura method). And on agitation with water an abundant amount of substances containing phosphorus and hexosamine came out in a fine suspended state. They could not be separat-

,ed by centrifugation but could be filtered off on paper pulp by suction? and also subsided on addition of $\mathrm{NaCl}$ up to $5 \%^{71}$ or of acetic acid. The precipitates only swelled without dissolution in alkaline $\left(\mathrm{Na}_{2} \mathrm{CO}_{3}\right)$ water. It involved $4.5 \%$ hexosamine, but many re-depositions after taking up as a suspension in the alkaline water by means of $\mathrm{HCl}$ (added to $\mathrm{pH}$ of 2.0 ) as well as fractionation at different $\mathrm{pH}$ 's neither raised the hexosamine content nor eliminated phosphorus. The nature of the hexosamine compound here has not been followed up. 

anymore.

Extracted with water, the residue showed no hexosamine reaction Biological Assays.

Hemopoietic and hemolytic activities, effect on blood coagulation and toxicity were examined.

Hemopoietic activity. Frs. $A_{1 \mathrm{a}}$ and $\mathrm{A}_{2}$ showed to possess this property.

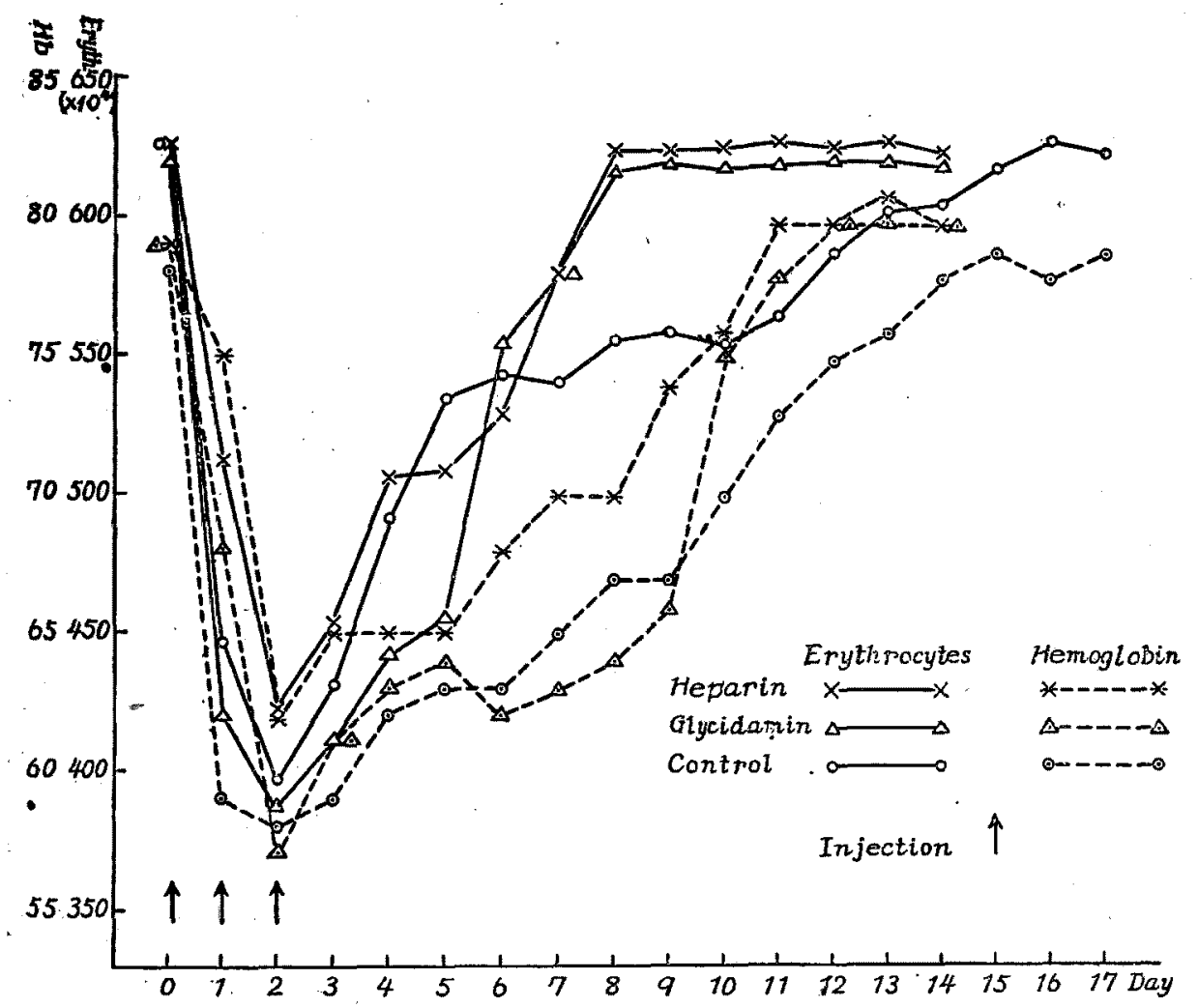

Fig. 1. Effect of heparin and the hemopoietic glycidamin from lung on the recovery of erythrocyte number and hemoglobin content of rabbits after bloodletting.

$15 \mathrm{cc}$. of blood per kgm: bodyweight were drawn from an ear vein of rabbits and after 1/2, 24 and 48 hours, $1 \mathrm{mgm}$. of the substances per kgm. bodyweight was injected intravenously as dissolved in the physiological saline solution ( $1 \mathrm{mgm}$. in $1 \mathrm{cc}$.). Five rabbits were employed for each of the substances and for the control runs where plain physiological saline was injected. In the controls it took more than two weeks for both erythrocyte number (Thoma-Zeiss) and hemoglobin content (Sahli) to resume the values before the bloodletting (the mean values in three successive days), but in the cases where the substances were applied, 8-9 and 8-12 days 
respectively were sufficient for the recovery. The curves plotted in Fig. 1 show the mean fluctuations of two experiments each. The experiments selected for the averaging were those on the rabbits all of which had closely. resembling normal values of hemoglobin content and erythrocyte number.

Effect on blood clotting. The clotting time was measured with an apparatus similar in design to the one by Chargaff, Bancroft and StanleyBrown. ${ }^{81}$ Into the glass tubes $(11 \times 75 \mathrm{~mm}$.) were pipetted $0.2 \mathrm{cc}$. of a solution diluted successively with physiological saline and then $0.5 \mathrm{cc}$. of human venous blood ( $B$ blood was used in this case) added, plunging the needle tip of the syringe to the bottom. The solution was substituted with simple physiological saline in one control and with $0.4 \%$ sodium citrate solution in physiological saline in the other. The temperature of the water thermostat was fixed at $20^{\circ} \mathrm{C}$. In the controls coagulation took place in $5^{\prime} 20^{\prime \prime}-5^{\prime} 30^{\prime \prime}$ and $22^{\prime}-25^{\prime}$ respectively after mixing the solution and blood together. Fr. $A_{1 a}$ (heparin) prolonged the clotting time to $31^{\prime}, 11^{\prime}$ and $6^{\prime}$ respectively at dilutions of $1: 200,000,1: 2,000,000$ and $1: 20,000,000$. Fr. $B_{1}$ only doubled the clotting time $\left(10^{\prime} 20^{\prime \prime}\right)$ at a dilution of $1: 100$ and Fr. $\mathrm{A}_{2}{ }^{9}$ ) had no effect on the clotting at all.

Hemolytic activity. To $0.2 \mathrm{cc}$. of a successively diluted solutions with physiological saline were added 2 drops of a $2 \%$ erythrocyte suspension (human $B$ erythrocytes were taken) and the mixtures kept in a water thermostat at $37^{\circ} \mathrm{C}$ for 2 hours. Neither Fr. $A_{2}{ }^{9}{ }^{\text {) }}$ nor Fr. $B_{1}$ developed this power even at only 1:200 dilution.

Group specifity (Table V). Isoagglutination of A erythrocytes was inhibited distinctly by Fr. $B_{1}$. The inhibition exhibited minutely by $\mathrm{Fr}$. $\mathrm{A}_{2}{ }^{9}$ ) is probably due to adhering Fr. $B_{1}$. The method was the one hitherto employed in this series of investigation. A and B sera used had agglutinin titers $\times 160$ and $\times 80$.

Poisonousness. $20 \mathrm{mgm}$. of Frs. A and B were applied for $10 \mathrm{gm}$. body-

TABLE V.

Occurrence of Isoagglutination of Erythrocytes, When the Sera are Preliminarily Treated with Fractions $A_{2}$ and $B_{1}$.

\begin{tabular}{|c|c|c|c|c|c|c|}
\hline Fraction & $\underset{\substack{\text { Group of } \\
\text { serum }}}{D i l}$ & $\begin{array}{c}1 \% \\
\text { (Original) }\end{array}$ & $\times 10$ & $\times 10^{2}$ & $\times 10^{3}$ & $\times 10^{4}$ \\
\hline $\mathrm{B}_{1}$ & $\begin{array}{l}\mathrm{A} \\
\mathrm{B}\end{array}$ & $\ddot{H}$ & \# & \# & $\stackrel{\text { H }}{+}$ & H \\
\hline $\mathrm{A}_{2}$ & $\stackrel{A}{\mathrm{~A}}$ & $\#$ & + & $\begin{array}{l}\text { H } \\
H\end{array}$ & \# & \\
\hline
\end{tabular}


weight to a mouse intraperitoneally. But there appeared no poisoning symptom. For injection, Fr. B was dissolved immediately in physiological saline, but Fr. A was dissolved at first in water by addition of $5 \%$ $\mathrm{Na}_{2} \mathrm{CO}_{3}$ solution until neutral and an equal volume of $1.7 \% \mathrm{NaCl}$ added.

\section{SUMmary.}

1. Heparin and two glycidamins were isolated from the lung tissue of pig. One of the glycidamins contained hydrolysable sulfur..

2. Heparin and the hydrolysable sulfur-containing glycidamin proved hemopoietic.

3. The glycidamin devoid of hydrolysable sulfur inhibited isoagglutination of A erythrocytes specifically.

The costs of this work were covered by a grant by the Grant Committee for Scientific Researches of the Education Department, which is gratefully acknowledged.

\section{Bibliography and Notes.}

1) The precipitate was small.

2) Further extraction was given up, because the products in similar runs had been found of much less amount and, in addition, were mostly insoluble in glycol at the initial stage of subsequent fractionation.

3) This was very small.

4) The remaining unextracted gave biuret reaction intensively but Molisch reaction barely.

5) $a$-Naphthol-sulfuric acid test resulted as in the case of Fr. $A_{1 b}$, namely this was revealed to still involve a glycidamin or a carbohydrate.

6) No precipitation occurred on further repetition of changing the $\mathrm{pH}$.

7) From the filtrate and the supernatant liquid could be obtained alcohol-precipitable ubstances after condensation and after dialysis and condensation respectively, but they amounted very small and moreover gave almost no reaction with Molisch reagents.

8) Chargaff, Bancroft and Stanley-Brown, J. Biol. Chem., 1936, 115, 149.

9) The original solution was prepared by taking up the substance in physiological saline followed by neutralizing with $5 \% \mathrm{Na}_{2} \mathrm{CO}_{3}$. 